\title{
Targeting F508del-CFTR to develop rational new therapies for cystic fibrosis
}

\author{
Zhi-wei CAI, Jia LIU, Hong-yu LI, David N SHEPPARD* \\ School of Physiology and Pharmacology, University of Bristol, Medical Sciences Building, University Walk, Bristol BS8 1TD, UK
}

\begin{abstract}
The mutation F508del is the commonest cause of the genetic disease cystic fibrosis (CF). CF disrupts the function of many organs in the body, most notably the lungs, by perturbing salt and water transport across epithelial surfaces. F508del causes harm in two principal ways. First, the mutation prevents delivery of the cystic fibrosis transmembrane conductance regulator (CFTR) to its correct cellular location, the apical (lumen-facing) membrane of epithelial cells. Second, F508del perturbs the $\mathrm{Cl}^{-}$channel function of CFTR by disrupting channel gating. Here, we discuss the development of rational new therapies for CF that target F508del-CFTR. We highlight how structural studies provide new insight into the role of F508 in the regulation of channel gating by cycles of ATP binding and hydrolysis. We emphasize the use of high-throughput screening to identify lead compounds for therapy development. These compounds include CFTR correctors that restore the expression of F508del-CFTR at the apical membrane of epithelial cells and CFTR potentiators that rescue the F508del-CFTR gating defect. Initial results from clinical trials of CFTR correctors and potentiators augur well for the development of small molecule therapies that target the root cause of CF: mutations in CFTR.
\end{abstract}

Keywords: ATP-binding cassette transporter; epithelial ion transport; cystic fibrosis; CFTR; chloride ion channel; F508del; CFTR corrector; CFTR potentiator

Acta Pharmacologica Sinica (2011) 32: 693-701; doi: 10.1038/aps.2011.71

\section{Introduction}

Salty sweat is diagnostic of cystic fibrosis (CF), an autosomal recessive genetic disease common in Caucasians ${ }^{[1,2]}$. The elevated concentration of salt in sweat is indicative of the underlying molecular defect in $\mathrm{CF}$, the loss of chloride ion $\left(\mathrm{Cl}^{-}\right)$channel function in the apical (lumen-facing) membrane of epithelia lining ducts and tubes throughout the body ${ }^{[1]}$. The impermeability of the apical membrane to $\mathrm{Cl}^{-}$in $\mathrm{CF}$ disrupts fluid and electrolyte transport across epithelia and, hence, the function of a variety of organs. This leads to the wide-ranging manifestations of the disease, which include chronic lung disease, exocrine pancreatic insufficiency, meconium ileus (blockage of the terminal ileum), male infertility and salty sweat ${ }^{[1,}$ 2]. The median survival of CF patients in North America and Western Europe is around 40 years ${ }^{[2]}$.

There are two principal causes of debilitation and death in CF patients ${ }^{[1,3,4]}$. First, chronic obstructive lung disease caused by thick tenacious mucus that prevents normal mucociliary clearance. Second, persistent bacterial infections, typically

\footnotetext{
* To whom correspondence should be addressed.

E-mail D.N.Sheppard@bristol.ac.uk

Received 2011-03-27 Accepted 2011-04-25
}

with Pseudomonas aeruginosa, which result in bronchiectasis, respiratory failure and eventually death. Current therapies for CF include physiotherapy, mucolytic drugs and antibiotics to treat lung disease, and pancreatic enzyme replacement and supplementary nutrition to overcome gastrointestinal dysfunction $^{[1,2]}$. These therapies treat the symptoms of CF; they do not target the root cause of the disease.

In 1989, the defective gene responsible for CF was identified and predicted to encode a protein with five domains: two membrane-spanning domains (MSDs), two nucleotidebinding domains (NBDs) and a unique regulatory domain $(\mathrm{RD})^{[5]}$. Shortly thereafter, the protein product of this gene, the cystic fibrosis transmembrane conductance regulator (CFTR), was demonstrated to be a unique member of the ATP-binding cassette $(A B C)$ transporter superfamily ${ }^{[6]}$. Instead of forming an ATP-driven pump like most family members, CFTR was demonstrated to function as an ATP-gated pathway for anion movement driven by the transmembrane electrochemical gradient ${ }^{[7-10]}$. Subsequent research has aimed to understand the physiological role of CFTR, learn how CF mutations cause CFTR dysfunction and develop rational new therapies for CF patients. Here, we selectively review progress in the development of drug therapies for $\mathrm{CF}$, focusing on small molecules 
that target the most common CF mutation, F508del, deletion of the three base pairs that result in the loss of the phenylalanine residue at position 508 of the CFTR protein sequence; $90 \%$ of CF patients carry at least one copy of the F508del mutation.

\section{The F508del-CFTR mutant retains some residual channel function}

When Rich et al ${ }^{[11]}$ found that expression of F508del-CFTR in $\mathrm{CF}$ airway epithelial cells failed to correct the defective $\mathrm{Cl}^{-}$permeability of these cells and Cheng et al ${ }^{[12]}$ subsequently demonstrated that the F508del mutation disrupts CFTR biosynthesis and membrane trafficking in COS-7 cells, it was widely assumed that F508del-CFTR had no $\mathrm{Cl}^{-}$channel function. Surprisingly however, Drumm et al ${ }^{[13]}$ demonstrated that F508delCFTR generates a cAMP-activated $\mathrm{Cl}^{-}$conductance when expressed in Xenopus oocytes. Because F508del-CFTR $\mathrm{Cl}^{-}$currents had similar conduction and permeation properties, but reduced magnitude compared with those of wild-type CFTR, Drumm et al ${ }^{[13]}$ speculated that F508del-CFTR forms a channel with attenuated sensitivity to cAMP agonists, a conclusion that was to prove prescient. Concurrently, Dalemans et al ${ }^{[14]}$ used the patch-clamp technique to demonstrate that F508delCFTR forms a $\mathrm{Cl}^{-}$channel regulated by cAMP-dependent phosphorylation in Vero cells. The authors demonstrated that F508del-CFTR had many properties in common with those of wild-type human $\mathrm{CFTR}^{[14]}$. However, there was one notable exception, the pattern of channel gating of F508del-CFTR differed dramatically from that of wild-type $\mathrm{CFTR}^{[14]}$.

\section{Biophysical properties of the F508del-CFTR $\mathrm{Cl}^{-}$channel}

Like other mutations that affect specific residues within the $\mathrm{NBDs}^{[15]}$, F508del has no discernable effect on the conduction and permeation properties of the CFTR $\mathrm{Cl}^{-}$channel. First, the F508del-CFTR $\mathrm{Cl}^{-}$channel has a small single-channel conductance, which does not differ from that of wild-type CFTR (6-10 pS; eg Li et al ${ }^{[16]}$ ). Second, like wild-type CFTR (but see ${ }^{[17]}$ ), the current-voltage (I-V) relationship of F508del-CFTR is linear (eg Dalemans et $\left.\mathrm{al}^{[14]}\right)$. Third, both wild-type and F508del-CFTR are highly selective for anions over cations $\left(\mathrm{P}_{\mathrm{Na}} / \mathrm{P}_{\mathrm{Cl}}=0.08 ; e g \mathrm{Li}\right.$ et $\left.\mathrm{al}^{[16]}\right)$. Fourth, wild-type and F508del-CFTR share the identical anion permeability sequence of $\mathrm{Br}^{-}>\mathrm{Cl}^{-}>\mathrm{I}^{-}$(eg Dalemans et $\left.a l^{[14]}\right)$. Finally, wild-type and F508del-CFTR both exhibit timeand voltage-independent gating behavior (eg Denning et al ${ }^{[18]}$ ). Consistent with these data, using excised membrane patches from gallbladder epithelial cells of wild-type and F508delCFTR mice French et al ${ }^{[19]}$ demonstrated that the F508del mutation is without effect on the biophysical properties of murine CFTR. Thus, the data suggest that the F508del mutation does not affect the pore properties of CFTR.

\section{The gating defect of the F508del-CFTR $\mathrm{Cl}^{-}$channel}

Figure 1 illustrates the gating pattern of wild-type and F508del-CFTR $\mathrm{Cl}^{-}$channels following phosphorylation by protein kinase A (PKA). The gating behavior of wild-type CFTR is characterized by frequent bursts of channel activity that are interrupted by brief flickery closures and separated by longer closures between bursts (Figure 1). By contrast, the gating pattern of F508del-CFTR is characterized by infrequent bursts of channel activity that are interrupted by brief flickery closures, but separated by long closures of prolonged duration (Figure 1). Work by a number of investigators using a variety of cells and experimental conditions demonstrate that the open probability $\left(\mathrm{P}_{\mathrm{o}}\right.$; a measure of the average fraction of time that a channel is open) of F508del-CFTR is about one third that of wild-type CFTR $^{[14,18,20-23]}$, although Miki et al ${ }^{[24]}$ argue that the Po of F508del-CFTR is likely to be substantially lower ( $\sim$ fifteen-fold less than that of wild-type CFTR). Surprisingly, and in marked contrast to these data, other authors found that the $P_{o}$ of F508del-CFTR did not differ from that of wild-type $\mathrm{CFTR}^{[16,19]}$. A likely explanation for these differences is that the rate of activation of F508del-CFTR is more than seven-fold slower than that of wild-type CFTR ${ }^{[25]}$.
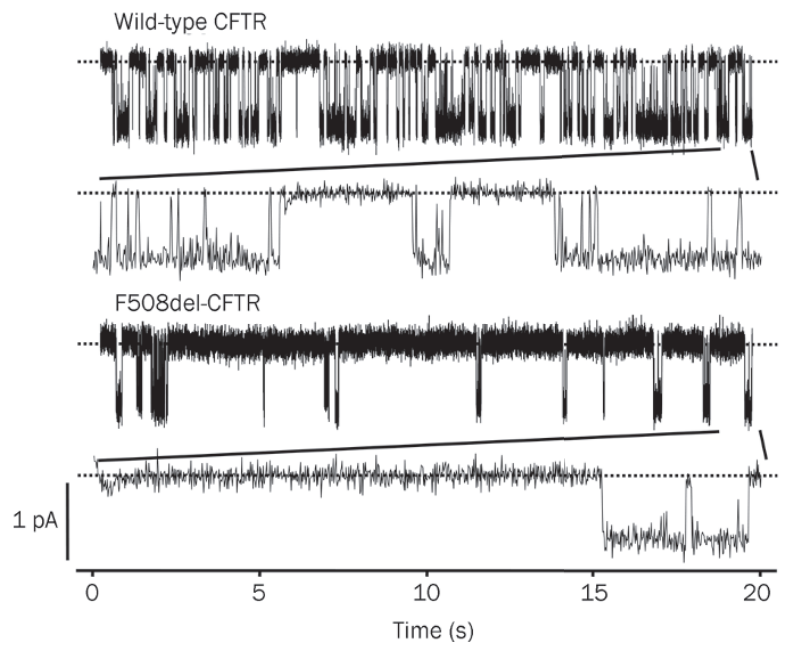

Figure 1. Single-channel activity of wild-type and F508del-CFTR. Representative recordings of wild-type and F508del-CFTR $\mathrm{Cl}^{-}$channels in excised inside-out membrane patches from $\mathrm{C} 127$ cells expressing recombinant CFTR. ATP $(1 \mathrm{mmol} / \mathrm{L})$ and PKA $(75 \mathrm{nmol} / \mathrm{L})$ were continuously present in the intracellular solution, voltage was clamped at $-50 \mathrm{mV}$, and a large $\mathrm{Cl}^{-}$concentration gradient was imposed across the membrane patch $\left(\left[\mathrm{Cl}^{-}\right]_{\mathrm{Ext}}=10 \mathrm{mmol} / \mathrm{L} ;\left[\mathrm{Cl}^{-}\right]_{\mathrm{Int}}=147 \mathrm{mmol} / \mathrm{L}\right)$. Dashed lines indicate where the channels are closed and downward deflections correspond to channel openings. Beneath each of the prolonged $20 \mathrm{~s}$ recordings, the last $1 \mathrm{~s}$ of the record is shown on an expanded scale. Other details are as described in Cai and Sheppard ${ }^{[23]}$. Modified, with permission, from Cai and Sheppard ${ }^{[23]}$.

To understand how the F508del mutation disrupts CFTR channel gating, several investigators have examined the gating kinetics of the F508del-CFTR $\mathrm{Cl}^{-}$channel. Dalemans et $a l^{[14]}$ first demonstrated that in cell-attached membrane patches on Vero cells voltage-clamped at $-60 \mathrm{mV}$, the F508del mutation was without effect on open times, but decreased mean closed times five-fold compared with those of wild-type CFTR. Haws et al ${ }^{[20]}$ and our group ${ }^{[23,26]}$ examined the gating kinetics of F508del-CFTR in membrane patches from BHK 
and $\mathrm{C} 127$ cells. Both groups found that the F508del mutation was without effect on open and closed times within bursts. Instead, F508del caused a large decrease in $\mathrm{P}_{\mathrm{o}}$ by (i) markedly prolonging the closed time interval between bursts and (ii) reducing mean burst duration ${ }^{[2,23,26]}$. These data suggest that the F508del mutation disrupts CFTR channel gating in two ways: first, F508del dramatically slows the rate of entry into a burst of channel activity. Second, F508del accelerates the rate of channel closure.

\section{F508del is located at a critical interface in the CFTR gating pathway}

The F508del mutation profoundly disrupts CFTR channel gating by slowing dramatically the rate of channel opening and by accelerating the rate of channel closure. An explanation for the gating behavior of F508del-CFTR is provided by the ATPdriven NBD dimerization model of CFTR channel gating ${ }^{[27,28]}$. This model integrates the results of functional studies of CFTR channel gating with biochemical and structural data. Structural studies of $\mathrm{ABC}$ transporters suggest that the NBDs are organized as a head-to-tail dimer with two ATP-binding sites located at the NBD1:NBD2 interface ${ }^{[2-32]}$. The data suggest that one ATP-binding site is formed by the Walker A and B motifs of NBD1 and the LSGGQ motif of NBD2 (termed site 1 ), while the other is formed by the Walker A and B motifs of NBD2 and the LSGGQ motif of NBD1 (termed site 2) (Figure 2). However, photolabeling studies argue that the ATP-binding sites of CFTR are not equivalent in function; site 1 stably binds nucleotides, whereas site 2 rapidly hydrolyses them ${ }^{[33,34]}$. Because CFTR $\mathrm{Cl}^{-}$channels transit between the closed and open configurations in seconds, Vergani et al ${ }^{[27,28]}$ interpreted the photolabeling data to suggest that CFTR channel gating is controlled by ATP binding and hydrolysis at site 2, driving cycles of NBD dimer assembly and disassembly. To test this model, Vergani et al ${ }^{[28]}$ applied mutant cycle analysis to residues predicted to lie on opposite sides of the NBD1:NBD2 dimer interface. Of note, the authors demonstrated that R555 (NBD1) and T1246 (NBD2) are energetically coupled only in open channels, arguing convincingly that the NBDs undergo dynamic reorganization during channel gating ${ }^{[28]}$. For further discussion of how ATP gates the CFTR $\mathrm{Cl}^{-}$channel, see ${ }^{[35-37]}$.

Using the ATP-driven NBD dimerization model of CFTR channel gating ${ }^{[27,28]}$, Roxo-Rosa et al ${ }^{[26]}$ speculated that the exceptionally slow rate of channel opening of F508del-CFTR might be explained by F508del inducing misfolding and/ or structural instability of NBD1, which would hamper ATP binding. Moreover, the reduced open time of F508del-CFTR might reflect weakening of the binding energy for stable NBD1:NBD2 dimer formation by the mutation ${ }^{[26]}$. In support of this idea, Pissarra et al ${ }^{[38]}$ demonstrated that the solubilizing mutations used to promote crystallization of human NBD $1^{[32]}$ traffic F508del-CFTR to the surface and abrogate, albeit incompletely, the channel's gating defect. Thus, deletion of F508 might cause intrinsic misfolding and/or structural instability of NBD1 ${ }^{[26]}$.

However, two lines of evidence argue against the idea that deletion of F508 causes misfolding of NBD1. First, F508del perturbs the local topography of NBD1, without affecting domain folding ${ }^{[32]}$, but see Pissarra et al ${ }^{[38]}$. Second, F508 is located at the surface of NBD1, where it might interact with the MSDs ${ }^{[31,32]}$. The residue is remote from the NBD1:NBD2 interface, the location of the ATP-binding sites (Figure 2).

Following the elucidation of the atomic structure of the ABC transporter Sav1866, the multidrug transporter of $S$ aureus ${ }^{[39]}$,

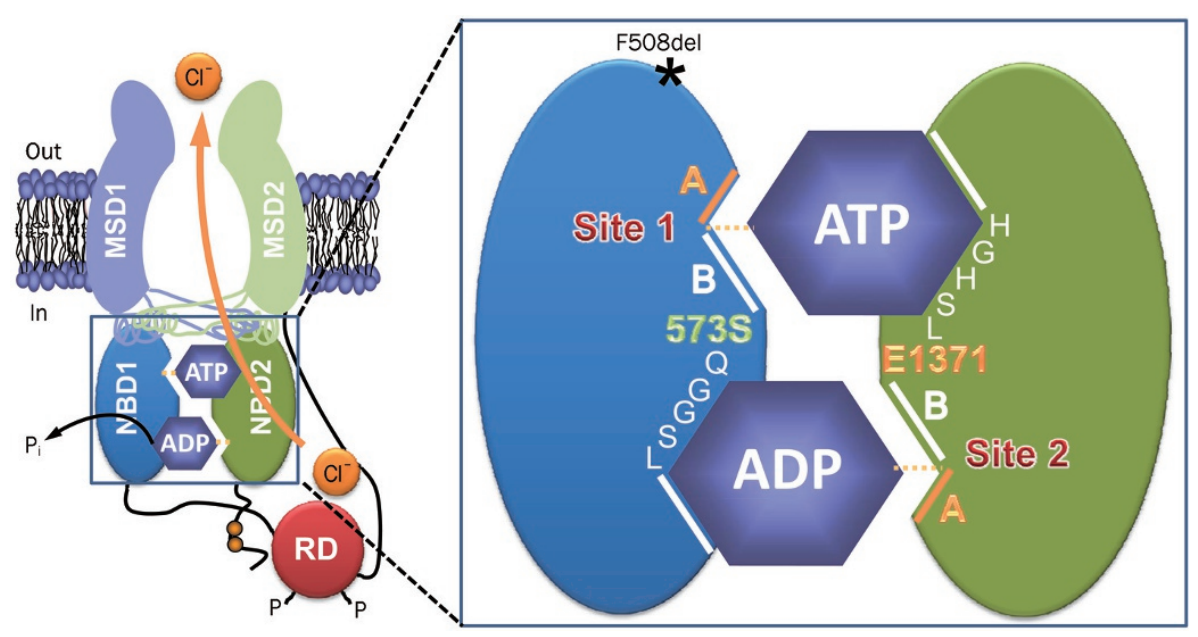

Figure 2. The organization of the ATP-binding sites in CFTR. The simplified model shows the molecular architecture of ATP-binding site 1 (site 1) and ATP-binding site 2 (site 2) in an open CFTR Cl- channel. Each ATP-binding site is formed by the Walker A and B motifs (labeled A and B, respectively) of one NBD and the LSGGQ motif of the other NBD. Site 2 contains a canonical LSGGQ motif, whereas site 1 contains a non-canonical LSGGQ motif (LSHGH). Site 2 also contains a catalytic base (E1371) at the distal end of the Walker B motif, but this residue is absent in site 1 (S573). The location of the CF mutation F508del on the surface of NBD1 opposite intracellular loop 4 (ICL4) is shown by an asterisk. Abbreviations: MSD, membrane-spanning domain; NBD, nucleotide-binding domain; P, phosphorylation of the RD; $\mathrm{P}_{\mathrm{i}}$, inorganic phosphate; RD, regulatory domain. In and out denote the intraand extracellular sides of the membrane, respectively. See text for further information. Modified, with permission, from Hwang and Sheppard ${ }^{[36]}$. 
structural models of the entire CFTR protein have been developed ${ }^{[40-42]}$ and used to understand better CFTR function (eg Alexander et $a l^{[43]}$ ). Of note, these structural models have provided important new insight into how the F508del mutation disrupts CFTR channel gating. They also reveal the function of the four intracellular loops (ICLs), which connect transmembrane segments within the MSDs. Each ICL consists of two long a-helical extensions of transmembrane segments with an intervening short a-helix at its most cytoplasmic location orientated parallel to the plane of the membrane. Because this short a-helix interacts with the NBDs, it is termed the coupling helix ${ }^{[40,41]}$.

For two reasons, the positions of the ICLs in structural models of CFTR are notable. First, the ICLs communicate both with the same and the opposite NBD (eg ICL1 (MSD1) with NBD1 and ICL2 (MSD1) with NBD2 $2^{[40,41]}$ ). Prior to these structural models, communication between the NBDs and MSDs was presumed to be only vertical (eg NBD1:MSD1). However, the structural models of Serohijos et al ${ }^{[40]}$ and Mornon et al ${ }^{[41]}$ argue that communication between the NBDs and MSDs is both vertical and orthogonal (eg NBD1:MSD1 and NBD1:MSD2). Second, the coupling helix of ICL4 interacts with the surface of NBD1 in the region of F508 ${ }^{[40,41]}$. This observation provides an explanation for why the F508del mutation profoundly disrupts CFTR channel gating. The mutation affects a residue located at a critical interface in the CFTR gating pathway, the sequence of conformation changes initiated by ATP-driven NBD dimerization, which leads to opening of the CFTR pore and $\mathrm{Cl}^{-}$flow through the channel. Thus, understanding the interface between the F508 region of NBD1 and the coupling helix of ICL4 is central to the development of drug therapies that target the root cause of CF.

\section{Rational new therapies for CF that target defects in F508del-CFTR}

To target the root cause of CF, future therapies should (i) overcome the F508del-CFTR processing defect and traffic the mutant protein to the apical membrane ${ }^{[12]}$; (ii) extend the residence time of F508del-CFTR at the apical membrane ${ }^{[44]}$ and abrogate channel "rundown" (eg Schultz et al ${ }^{[22]}$ ) and (iii) rescue the defective channel gating of F508del-CFTR ${ }^{[14]}$. Thus, small molecules with two or possibly three types of activity are required to restore function to the F508del-CFTR $\mathrm{Cl}^{-}$channel.

Small molecules that overcome the processing defect of F508del-CFTR and traffic the mutant protein to the apical membrane are termed CFTR correctors because they rescue the cell surface expression of F508del-CFTR ${ }^{[4,46]}$. CFTR correctors might interact with CFTR itself, by acting as either substrate mimics or active site inhibitors. Alternatively, they might target one or more of the many CFTR interacting proteins that orchestrate and control processing of CFTR, its delivery to, and expression at the apical membrane. This latter group of CFTR correctors is termed proteostasis regulators because they aim to treat $\mathrm{CF}$ by manipulating the concentration, conformation, quaternary structure and/or location of $\mathrm{CFTR}^{[47]}$.
Small molecules that repair the gating defect of the F508delCFTR $\mathrm{Cl}^{-}$channel are termed CFTR potentiators because they do not open silent channels, but instead enhance ATPdependent channel gating following the phosphorylation of F508del-CFTR by PKA ${ }^{[45]}$. Although some agents (eg bromotetramisole ${ }^{[48]}$ ) enhance CFTR gating by modulating activity of the protein kinases and phosphatases that control the phosphorylation status of CFTR, CFTR potentiators interact directly with CFTR to enhance channel gating. Interestingly, a small number of compounds have been identified which possess both CFTR corrector and potentiator activity ${ }^{[49,50]}$. These small molecules are termed CFTR corrector-potentiators or dual-acting molecules.

Because there is insufficient information at the present time to design rationally CFTR correctors and potentiators, the strategy of choice to identify drug-like small molecule CFTR modulators is high-throughput screening (HTS) ${ }^{[45]}$. HTS exploits a reliable, sensitive, cost-effective assay to screen libraries of chemically diverse small molecules (eg approved drugs $^{[51]}$, drug-like chemicals ${ }^{[52]}$ and natural products ${ }^{[53]}$ ) to identify lead compounds for medicinal chemistry optimization. For example, Alan Verkman (UCSF, San Francisco, USA) used Fischer rat thyroid cells, epithelial cells devoid of CFTR expression and cAMP-stimulated $\mathrm{Cl}^{-}$currents ${ }^{[54]}$ engineered to co-express recombinant human CFTR and a green fluorescent protein (GFP) with ultra high halide sensitivity in a halide flux assay (eg Yang et $a l^{[52]}$ ). By contrast, Vertex Pharmaceuticals (San Diego, USA) employed NIH-3T3 cells expressing recombinant human CFTR in a fluorescence resonance energy transfer (FRET)-based membrane voltage-sensing assay (eg Van Goor et $\left.a l^{[55]}\right)$. Both of these HTS assays monitor the change in CFTR-mediated anion flux elicited by CFTR modulators in real time.

By screening 150000 drug-like compounds using F508delCFTR expressing FRT cells, Verkman's group were the first to identify CFTR correctors using HTS ${ }^{[56]}$. Among the chemical scaffolds with CFTR corrector activity, the bisaminomethylbithiazole corr-4a (Figure 3) deserves special attention. Pedemonte et $a l^{[56]}$ demonstrated that corr-4a is equipotent to low temperature correction at restoring function to F508del-CFTRexpressing human bronchial epithelia (CFBE), achieving levels of CFTR function approximately $8 \%$ of that of human bronchial epithelia expressing wild-type CFTR (HBE). Of special note, the aminoarylthiazole corr- $2 \mathrm{~b}$ identified by Pedemonte et $a l^{[56]}$ exhibits dual activity as both a CFTR corrector and a CFTR potentiator ${ }^{[50]}$. When compared with small molecules that act as CFTR correctors, corr- $2 \mathrm{~b}$ generated double the amount of forskolin-activated $\mathrm{CFTR} \mathrm{Cl}^{-}$current $\left(I_{\mathrm{FSK}}\right)$ relative to the total $\mathrm{CFTR}^{-}$current measured in the presence of forskolin and the CFTR potentiator genistein $\left(\mathrm{I}_{\mathrm{TOT}}\right)$ (CFTR correctors (eg corr- $4 \mathrm{a}): I_{\mathrm{FSK}} / I_{\mathrm{TOT}} \sim 40 \%$; corr $-2 \mathrm{~b}: I_{\mathrm{FSK}} / I_{\mathrm{TOT}} \sim 80 \%$; see Figure 1 of Pedemonte et $\left.a l^{[50]}\right)$. Moreover, the authors demonstrated that aminoarylthiazoles do not act as typical CFTR potentiators because they require protein synthesis to exert their effects (see below and ${ }^{[50]}$ ). Elucidation of the mechanism of action of corr- $2 b$ and related dual-acting small molecules is 


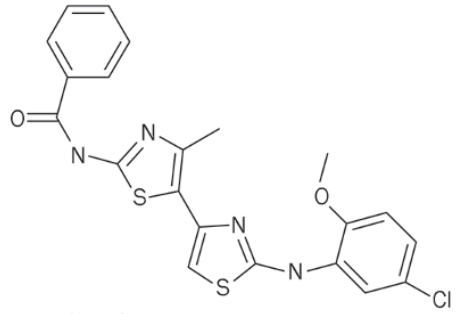

Corr-4a

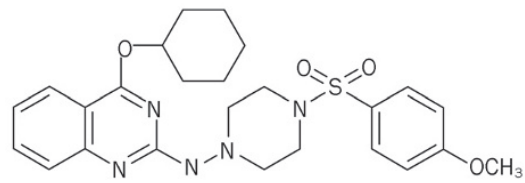

VRT-325

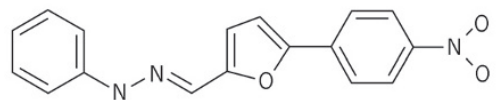

RDR1

Figure 3. Chemical structures of some CFTR correctors identified by HTS. Abbreviations: Corr-4a, N-[2-(5-Chloro-2-methoxy-phenylamino)-4'methyl-[4,5']bithiazolyl-2'-yl]-benzamide; VRT-325, 4-Cyclohexyloxy-2-\{1[4-(4-methoxy-benzensulfonyl)-piperazin-1-yl]-ethyl\}-quinazoline; RDR1, 5-(4-nitrophenyl)-2-furaldehyde 2-phenylhydrazone.

a priority for future research.

In a ground-breaking program funded by the Cystic Fibrosis Foundation (Bethesda, USA), a nonprofit, donor-supported organization, Vertex Pharmaceuticals identified 13 distinct chemical scaffolds with CFTR corrector activity after screening $\sim 164000$ chemically diverse drug-like small molecules ${ }^{[55]}$. Following medicinal chemistry optimization, Vertex Pharmaceuticals identified the quinazoline VRT-325 (Figure 3) as a potent and efficacious CFTR corrector that enhances the maturation of native F508del-CFTR protein and augments CFTR-mediated transepithelial $\mathrm{Cl}^{-}$secretion in $\mathrm{CFBE}^{[55]}$. Biochemical studies of VRT-325 suggest that it acts at the endoplasmic reticulum to promote CFTR folding ${ }^{[55]}$. Because VRT-325 decreases the apparent ATP affinity of purified, reconstituted F508del$\mathrm{CFTR}^{[57]}$, it might rescue the processing and trafficking of F508del-CFTR, at least in part, by interacting directly with the mutant protein.

To identify CFTR correctors that interact directly with F508del-CFTR, Sampson et al ${ }^{[58]}$ employed differential scanning fluorimetry, which identifies ligands of a target protein by monitoring their effects on the thermal unfolding of the protein. Among 224 hits identified in a previous HTS for CFTR correctors, just one chemical, the substituted phenylhydrazone RDR1 (Figure 3), was able to thermally stabilize murine F508del-CFTR ${ }^{[58]}$. As with previous studies of CFTR correctors by the Hanrahan and Thomas groups ${ }^{[59,60]}$, the authors deployed a battery of biochemical and functional assays to investigate F508del-CFTR rescue by RDR1 in heterologous cells, polarized epithelia and genetically-modified mice. The authors' data demonstrate that RDR1 thermally stabilizes murine F508del-NBD1, increases the maturation of human F508del-CFTR protein and augments the function of human CFTR in vitro and murine CFTR in vivo ${ }^{[58]}$. Taken together, these data and the additive effect of RDR1 treatment and low temperature incubation on human F508del-CFTR maturation argue convincingly that RDR1 is a CFTR corrector that targets directly F508del-NBD1 to exert its effects. Identification of the RDR1-binding site on CFTR should be an important goal of future research.

To identify CFTR potentiators that rescue the gating defect of F508del-CFTR, Yang et al ${ }^{[52]}$ studied FRT cells expressing low temperature-corrected F508del-CFTR. A screen of 100000 compounds identified six novel classes of highaffinity F508del-CFTR potentiators ${ }^{[52]}$. However, by screening additional structural analogues, Yang et al ${ }^{[52]}$ discovered tetrahydrobenzothiophenes (eg $\Delta \mathrm{F} 508_{\text {act }}-02$; Figure 4$)$, which potentiate F508del-CFTR with $K_{d}<100 \mathrm{nmol} / \mathrm{L}$. Subsequently, Pedemonte $e a^{[61]}$ screened 50000 compounds searching for further ligands that rescue the gating defect of F508del-CFTR. After secondary analyses, Pedemonte et al ${ }^{[61]}$ identified phenylglycines and sulfonamides that potentiate F508del-CFTR with nanomolar potency. Interestingly, by screening a library of 2000 compounds, including drugs approved for clinical use, Pedemonte et $a l^{[51]}$ demonstrated that the antihypertensive drugs 1,4-dihydropyridines (DHPs) act as F508del-CFTR potentiators by a mechanism independent of their effects on voltage-gated $\mathrm{Ca}^{2+}$ channels. To identify DHPs that potentiate F508del-CFTR without inhibiting voltage-gated $\mathrm{Ca}^{2+}$ channels, Pedemonte et $a l^{[62]}$ investigated structure-activity relationships

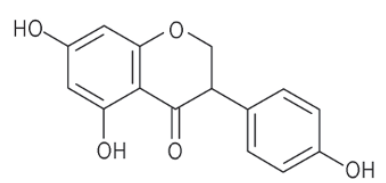

Genistein

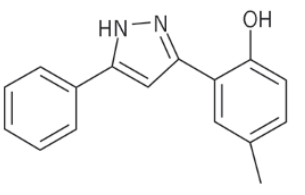

VRT-532

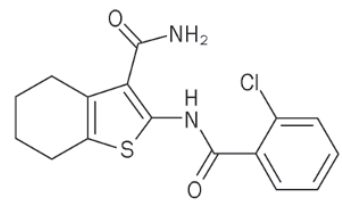

$\Delta \mathrm{F} 508_{\text {act }-02}$<smiles>CC(C)(C)c1cc(C(C)(C)C)c(NC(=O)c2c[nH]c3ccccc3c2=O)cc1O</smiles>

VX-770

Figure 4. Chemical structures of some CFTR potentiators identified by HTS. Abbreviations: $\Delta \mathrm{F} 508_{\text {act }}-02$, 2-(2-chlorobenzamido)-4,5,6,7tetrahydro-3H-indene-1-carboxamide; VRT-532, 4-methyl-2-(5-phenyl-1Hpyrazol-3-yl)phenol; VX-770, N-(2,4-di-tert-butyl-5-hydroxyphenyl)-4-oxo-1,4dihydroquinoline-3-carboxamide. For comparison, the chemical structure of genistein is shown. 
using a panel of 333 analogues of felodipine, the most potent CFTR potentiator identified in their original screen. The authors' data demonstrate that substituents with hydrophobic groups enhance the potency of DHPs as CFTR potentiators ${ }^{[2]}$. They also reveal that some DHPs are excellent lead compounds for the development of therapeutically active CFTR potentiators.

To identify therapeutically active potentiators of F508delCFTR, Vertex Pharmaceuticals screened 122000 synthetic compounds from their compound collection using NIH-3T3 cells expressing temperature-corrected F508del-CFTR ${ }^{[55]}$. After careful scrutiny, Van Goor et $a l^{[55]}$ selected for further study 53 compounds consisting of 10 distinct chemical scaffolds. One compound, the pyrazole VRT-532 (Figure 4), rescued the gating defect of F508del-CFTR by accelerating the rate of channel opening and slowing the rate of channel closure ${ }^{[55]}$. Critically, VRT-532 augmented robustly CFTR-mediated transepithelial $\mathrm{Cl}^{-}$secretion in CFBE $\left(\mathrm{EC}_{50}, 2.7 \pm 0.2 \mu \mathrm{mol} / \mathrm{L}^{[55]}\right)$. Importantly, the effects of VRT-532 on CFBE were synergistic with the CFTR corrector VRT-325. CFBE incubated with VRT-325 and then treated with CAMP agonists and VRT-532 generated levels of CFTR-mediated transepithelial $\mathrm{Cl}^{-}$secretion in CFBE $>20 \%$ of those observed in $\mathrm{HBE}^{[55]}$.

Subsequently, Vertex Pharmaceuticals screened 228000 chemically diverse drug-like compounds to identify chemical scaffolds for development into therapeutically active CFTR potentiators. Following medicinal chemistry optimization, Vertex Pharmaceuticals identified VX-770 (Figure 4), a potent, selective and orally bioavailable CFTR potentiator ${ }^{[63]}$. Interestingly, by increasing the frequency and duration of channel openings, VX-770 $(1 \mu \mathrm{mol} / \mathrm{L})$ restored the channel activity (measured by $\mathrm{P}_{\mathrm{o}}$ ) of F508del-CFTR to wild-type CFTR levels ${ }^{[63]}$. Moreover, treatment of CFBE (genotype F508del/G551D) with VX-770 $(10 \mu \mathrm{mol} / \mathrm{L})$ increased airway surface liquid volume and ciliary beat frequency to levels about half those of $\mathrm{HBE}^{[63]}$.

Based on its performance in preclinical studies, VX-770 became the first CFTR potentiator to be tested in the clinic. The drug was first tested in 39 adult CF patients carrying the CFTR mutation G551D, which has no effect on the processing and trafficking of CFTR, but profoundly disrupts channel gating $^{[64,65]}$. The CF patients in this study took VX-770 orally in a randomized, double-blind, placebo-controlled trial ${ }^{[66]}$. VX-770 was well tolerated by CF patients, and at high concentration $(150 \mathrm{mg}), \mathrm{VX}-770$ decreased the sweat $\mathrm{Cl}^{-}$concentration to a level approaching the normal range $(<60 \mathrm{mmol} / \mathrm{L})$ and improved lung function (measured by forced expiratory volume in one second, $\mathrm{FEV}_{1}$ ) by $9 \%{ }^{[66]}$. Further clinical studies of VX-770 are ongoing. Of special note, initial results from the phase III clinical trial of VX-770 on 83 CF patients with the G551D mutation demonstrated a sustained improvement in lung function at 48 weeks with drug-treated CF patients $55 \%$ less likely to experience a pulmonary exacerbation (http:// investors.vrtx.com/releasedetail.cfm?ReleaseID=551869). Vertex Pharmaceuticals indicate that they plan to apply for US and European drug approval later in 2011 (http:/ / investors. vrtx.com/releasedetail.cfm?ReleaseID=551869).
Knowledge of how ATP gates the CFTR $\mathrm{Cl}^{-}$channel, particularly the ATP-driven NBD dimerization model ${ }^{[27,28]}$, provides explanations for the mechanism(s) of action of CFTR potentiators. Ai et $a l^{[67]}$ first proposed that genistein and other CFTR potentiators might enhance CFTR channel gating by affecting NBD dimerization. The authors speculated first that the binding of genistein at the interface of the NBD dimer might lower the free energy of the transition state and, hence, accelerate channel opening ${ }^{[67]}$. Second, the authors proposed that genistein might slow the rate of channel closure by stabilizing the NBD dimer conformation ${ }^{[67]}$. Finally, the authors argued that the binding site for genistein might be located at the dimer interface ${ }^{[67]}$. Consistent with this idea, Moran et al ${ }^{[68]}$ used a molecular model of the NBD1:NBD2 dimer to show that genistein, apigenin and a series of novel CFTR potentiators identified by HTS bind to CFTR at the dimer interface. As predicted ${ }^{[69]}$ and verified ${ }^{[70]}$ by functional data, this drugbinding site is distinct from the two ATP-binding sites of CFTR. Moreover, sequences from both NBD1 (Walker A, Walker B and LSGGQ) and NBD2 (LSGGQ) contribute to the drug-binding site, with those from NBD1 forming a cavity in which CFTR potentiators dock ${ }^{[68]}$. Following the development of structural models of the entire CFTR protein ${ }^{[40-42]}$, in silico structure-based screening is likely to become a powerful tool to identify small molecules that interact directly with F508delCFTR and other CF mutants. Of note, using this approach Kalid et $a l^{[49]}$ identified a ligand-binding site in the vicinity of F508 at the interface of the NBDs and MSDs. Finally, differences in the molecular pharmacology of CFTR homologs from different species (eg human and murine $\operatorname{CFTR}^{[63,71,72]}$ ) argue that chimeric CFTR proteins may be valuable tools to identify where CFTR potentiators dock with $\mathrm{CFTR}^{[73]}$.

\section{Conclusions}

Two decades after the identification of the defective gene responsible for $\mathrm{CF}$, therapies based on a molecular understanding of the disease are beginning to be tested in the clinic. Early results from these trials are encouraging. They raise the prospect of personalized medicine, whereby specific therapies are designed to target precisely the genetic defects harbored by individuals afflicted by $\mathrm{CF}$. However, the development of efficacious and safe drug therapies for CF patients will require much more work. For example, it is currently unknown how much CFTR function is required to rescue CF mutants, whether drug therapy for $\mathrm{CF}$ is mutation specific and if longterm treatment with CFTR correctors and potentiators causes adverse effects. Answers to these pressing questions will play an important role in shaping future therapeutic strategies for CF.

\section{Acknowledgements}

We thank former laboratory colleagues for stimulating discussions. Work in the authors' laboratory is supported by the Cystic Fibrosis Trust and the Engineering and Physical Sciences Research Council [grant no. EP/F03623X/1]. J Liu is supported by scholarships from the University of Bristol and 
the Overseas Research Students Awards Scheme of Universities UK.

\section{Author contribution}

The authors researched the literature and wrote the review.

\section{References}

1 Welsh MJ, Ramsey BW, Accurso F, Cutting GR. Cystic fibrosis. In: Scriver CR, Beaudet AL, Sly WS, Valle D, editors. The Metabolic and Molecular Basis of Inherited Disease. New York: McGraw-Hill Inc; 2001. p 5121-88.

2 Davis PB. Cystic fibrosis since 1938. Am J Respir Crit Care Med 2006; 173: 475-82.

3 Rowe SM, Miller S, Sorscher EJ. Cystic fibrosis. N Engl J Med 2005; 352: 1992-2001.

4 Boucher RC. Airway surface dehydration in cystic fibrosis: pathogenesis and therapy. Annu Rev Med 2007; 58: 157-70.

5 Riordan JR, Rommens JM, Kerem B, Alon N, Rozmahel R, Grzelczak $Z$, et al. Identification of the cystic fibrosis gene: cloning and characterization of complementary DNA. Science 1989; 245: 106673.

6 Holland IB, Cole SPC, Kuchler K, Higgins CF. ABC Proteins: from bacteria to man. London: Academic Press; 2003.

7 Anderson MP, Gregory RJ, Thompson S, Souza DW, Paul S, Mulligan $\mathrm{RC}$, et al. Demonstration that CFTR is a chloride channel by alteration of its anion selectivity. Science. 1991; 253: 202-5.

8 Bear CE, Li CH, Kartner N, Bridges RJ, Jensen TJ, Ramjeesingh M, et al. Purification and functional reconstitution of the cystic fibrosis transmembrane conductance regulator (CFTR). Cell 1992; 68: 80918.

9 Anderson MP, Berger HA, Rich DP, Gregory RJ, Smith AE, Welsh MJ. Nucleoside triphosphates are required to open the CFTR chloride channel. Cell 1991; 67: 775-84.

10 Hwang TC, Nagel G, Nairn AC, Gadsby DC. Regulation of the gating of cystic fibrosis transmembrane conductance regulator $\mathrm{C} 1$ channels by phosphorylation and ATP hydrolysis. Proc Natl Acad Sci U S A 1994; 91: 4698-702.

11 Rich DP, Anderson MP, Gregory RJ, Cheng SH, Paul S, Jefferson DM, et al. Expression of cystic fibrosis transmembrane conductance regulator corrects defective chloride channel regulation in cystic fibrosis airway epithelial cells. Nature 1990; 347: 358-63.

12 Cheng SH, Gregory RJ, Marshall J, Paul S, Souza DW, White GA, et al. Defective intracellular transport and processing of CFTR is the molecular basis of most cystic fibrosis. Cell 1990; 63: 827-34.

13 Drumm ML, Wilkinson DJ, Smit LS, Worrell RT, Strong TV, Frizzell RA, et al. Chloride conductance expressed by $\Delta \mathrm{F} 508$ and other mutant CFTRs in Xenopus oocytes. Science 1991; 254: 1797-9.

14 Dalemans W, Barbry P, Champigny G, Jallat S, Dott K, Dreyer D, et al. Altered chloride ion channel kinetics associated with the $\Delta \mathrm{F} 508$ cystic fibrosis mutation. Nature 1991; 354: 526-8.

15 Sheppard DN, Welsh MJ. Structure and function of the CFTR chloride channel. Physiol Rev 1999; 79 (1 Suppl): S23-45.

16 Li C, Ramjeesingh M, Reyes E, Jensen T, Chang X, Rommens JM, et al. The cystic fibrosis mutation $(\Delta \mathrm{F} 508)$ does not influence the chloride channel activity of CFTR. Nat Genet 1993; 3: 311-6.

17 Cai Z, Scott-Ward TS, Sheppard DN. Voltage-dependent gating of the cystic fibrosis transmembrane conductance regulator $\mathrm{Cl}^{-}$channel. J Gen Physiol 2003; 122: 605-20.

18 Denning GM, Anderson MP, Amara JF, Marshall J, Smith AE, Welsh
MJ. Processing of mutant cystic fibrosis transmembrane conductance regulator is temperature-sensitive. Nature 1992; 358: 761-4.

19 French PJ, van Doorninck JH, Peters RH, Verbeek E, Ameen NA, Marino $\mathrm{CR}$, et al. A $\Delta \mathrm{F} 508$ mutation in mouse cystic fibrosis transmembrane conductance regulator results in a temperature-sensitive processing defect in vivo. J Clin Invest 1996; 98: 1304-12.

20 Haws CM, Nepomuceno IB, Krouse ME, Wakelee H, Law T, Xia Y, et al. $\triangle$ F508-CFTR channels: kinetics, activation by forskolin, and potentiation by xanthines. Am J Physiol 1996; 270: C1544-55.

21 Hwang TC, Wang F, Yang IC, Reenstra WW. Genistein potentiates wild-type and $\Delta$ F508-CFTR channel activity. Am J Physiol 1997; 273: C988-98.

22 Schultz BD, Frizzell RA, Bridges RJ. Rescue of dysfunctional $\Delta \mathrm{F} 508$ CFTR chloride channel activity by IBMX. J Membr Biol 1999; 170: 51-66.

23 Cai Z, Sheppard DN. Phloxine B interacts with the cystic fibrosis transmembrane conductance regulator at multiple sites to modulate channel activity. J Biol Chem 2002; 277: 19546-53.

24 Miki H, Zhou Z, Li M, Hwang TC, Bompadre SG. Potentiation of disease-associated cystic fibrosis transmembrane conductance regulator mutants by hydrolyzable ATP analogs. J Biol Chem 2010; 285: $19967-75$.

25 Wang F, Zeltwanger S, Hu S, Hwang TC. Deletion of phenylalanine 508 causes attenuated phosphorylation-dependent activation of CFTR chloride channels. J Physiol 2000; 524: 637-48.

26 Roxo-Rosa M, Xu Z, Schmidt A, Neto M, Cai Z, Soares CM, et al. Revertant mutants G550E and 4RK rescue cystic fibrosis mutants in the first nucleotide-binding domain of CFTR by different mechanisms. Proc Natl Acad Sci U S A 2006; 103: 17891-6.

27 Vergani P, Nairn AC, Gadsby DC. On the mechanism of MgATPdependent gating of CFTR $\mathrm{Cl}^{-}$channels. J Gen Physiol 2003; 121: 17-36.

28 Vergani P, Lockless SW, Nairn AC, Gadsby DC. CFTR channel opening by ATP-driven tight dimerization of its nucleotide-binding domains. Nature 2005; 433: 876-80.

29 Locher KP, Lee AT, Rees DC. The E. coli BtuCD structure: a framework for $A B C$ transporter architecture and mechanism. Science 2002; 296: 1091-8.

30 Smith PC, Karpowich N, Millen L, Moody JE, Rosen J, Thomas PJ, et al. ATP binding to the motor domain from an $A B C$ transporter drives formation of a nucleotide sandwich dimer. Mol Cell 2002; 10: 13949.

31 Lewis HA, Buchanan SG, Burley SK, Conners K, Dickey M, Dorwart M, et al. Structure of nucleotide-binding domain 1 of the cystic fibrosis transmembrane conductance regulator. EMBO J 2004; 23: 282-93.

32 Lewis HA, Zhao X, Wang C, Sauder JM, Rooney I, Noland BW, et al. Impact of the $\Delta F 508$ mutation in first nucleotide-binding domain of human cystic fibrosis transmembrane conductance regulator on domain folding and structure. J Biol Chem 2005; 280: 1346-53.

33 Aleksandrov L, Aleksandrov AA, Chang XB, Riordan JR. The first nucleotide binding domain of cystic fibrosis transmembrane conductance regulator is a site of stable nucleotide interaction, whereas the second is a site of rapid turnover. J Biol Chem 2002; 277: 15419-25.

34 Basso C, Vergani P, Nairn AC, Gadsby DC. Prolonged nonhydrolytic interaction of nucleotide with CFTR's $\mathrm{NH}_{2}$-terminal nucleotide binding domain and its role in channel gating. J Gen Physiol 2003; 122 333-48.

35 Gadsby DC, Vergani P, Csanády L. The ABC protein turned chloride channel whose failure causes cystic fibrosis. Nature 2006; 440 : 
477-83.

36 Hwang TC, Sheppard DN. Gating of the CFTR $\mathrm{Cl}^{-}$channel by ATPdriven nucleotide-binding domain dimerisation. J Physiol 2009; 587: 2151-61.

37 Kirk K, Wang W. A unified view of cystic fibrosis transmembrane conductance regulator (CFTR) gating: combining the allosterism of a ligand-gated channel with the enzymatic activity of an ATP-binding cassette (ABC) transporter. J Biol Chem 2011; 286: 12813-9.

38 Pissarra LS, Farinha CM, Xu Z, Schmidt A, Thibodeau PH, Cai Z, et al. Solubilizing mutations used to crystallize one CFTR domain attenuate the trafficking and channel defects caused by the major cystic fibrosis mutation. Chem Biol 2008; 15: 62-9.

39 Dawson RJ, Locher KP. Structure of a bacterial multidrug ABC transporter. Nature 2006 14; 443: 180-5.

40 Serohijos AW, Hegedus T, Aleksandrov AA, He L, Cui L, Dokholyan NV, et al. Phenylalanine-508 mediates a cytoplasmic-membrane domain contact in the CFTR 3D structure crucial to assembly and channel function. Proc Natl Acad Sci U S A 2008; 105: 3256-61.

41 Mornon JP, Lehn P, Callebaut I. Atomic model of human cystic fibrosis transmembrane conductance regulator: membrane-spanning domains and coupling interfaces. Cell Mol Life Sci 2008; 65: 2594-612.

42 Mornon JP, Lehn P, Callebaut I. Molecular models of the open and closed states of the whole human CFTR protein. Cell Mol Life Sci 2009; 66: 3469-86.

43 Alexander C, Ivetac A, Liu X, Norimatsu Y, Serrano JR, Landstrom $A$, et al. Cystic fibrosis transmembrane conductance regulator: using differential reactivity toward channel-permeant and channelimpermeant thiol-reactive probes to test a molecular model for the pore. Biochemistry 2009; 48: 10078-88.

44 Lukacs GL, Chang XB, Bear C, Kartner N, Mohamed A, Riordan JR, et al. The $\Delta \mathrm{F} 508$ mutation decreases the stability of cystic fibrosis transmembrane conductance regulator in the plasma membrane. Determination of functional half-lives on transfected cells. J Biol Chem 1993; 268: 21592-8.

45 Verkman AS, Galietta $\sqcup$. Chloride channels as drug targets. Nat Rev Drug Discov 2009; 8: 153-71.

46 Amaral MD. Targeting CFTR: How to treat cystic fibrosis by CFTRrepairing therapies. Curr Drug Targets. 2011; 12: 683-93.

47 Balch WE, Morimoto RI, Dillin A, Kelly JW. Adapting proteostasis for disease intervention. Science 2008; 319: 916-9.

48 Becq F, Jensen TJ, Chang XB, Savoia A, Rommens JM, Tsui LC, et al. Phosphatase inhibitors activate normal and defective CFTR chloride channels. Proc Natl Acad Sci U S A 1994; 91: 9160-4.

49 Kalid O, Mense M, Fischman S, Shitrit A, Bihler H, Ben-Zeev E, et al. Small molecule correctors of F508del-CFTR discovered by structurebased virtual screening. J Comput Aided Mol Des 2010; 24: 971-91.

50 Pedemonte N, Tomati V, Sondo E, Caci E, Millo E, Armirotti A, et al. Dual activity of aminoarylthiazoles on the trafficking and gating defects of the cystic fibrosis transmembrane conductance regulator (CFTR) chloride channel caused by cystic fibrosis mutations. J Biol Chem 2011; 286: 15215-26.

51 Pedemonte N, Diena T, Caci E, Nieddu E, Mazzei M, Ravazzolo R, et al. Antihypertensive 1,4-dihydropyridines as correctors of the cystic fibrosis transmembrane conductance regulator channel gating defect caused by cystic fibrosis mutations. Mol Pharmacol 2005; 68: 173646.

52 Yang H, Shelat AA, Guy RK, Gopinath VS, Ma T, Du K, et al. Nanomolar affinity small molecule correctors of defective $\triangle$ F508-CFTR chloride channel gating. J Biol Chem 2003; 278: 35079-85.

53 Xu LN, Na WL, Liu X, Hou SG, Lin S, Yang H, et al. Identification of natural coumarin compounds that rescue defective $\triangle$ F508-CFTR chloride channel gating. Clin Exp Pharmacol Physiol 2008; 35: 87883.

54 Sheppard DN, Carson MR, Ostedgaard LS, Denning GM, Welsh MJ. Expression of cystic fibrosis transmembrane conductance regulator in a model epithelium. Am J Physiol 1994; 266: L405-13.

55 Van Goor F, Straley KS, Cao D, González J, Hadida S, Hazlewood A, et al. Rescue of $\triangle F 508$-CFTR trafficking and gating in human cystic fibrosis airway primary cultures by small molecules. Am J Physiol Lung Cell Mol Physiol 2006; 290: L1117-30.

56 Pedemonte N, Lukacs GL, Du K, Caci E, Zegarra-Moran O, Galietta $\sqcup$, et al. Small-molecule correctors of defective $\triangle F 508$-CFTR cellular processing identified by high-throughput screening. J Clin Invest 2005; 115: 2564-71.

57 Kim Chiaw P, Wellhauser L, Huan LJ, Ramjeesingh M, Bear CE. A chemical corrector modifies the channel function of F508del-CFTR. Mol Pharmacol 2010; 78: 411-8.

58 Sampson HM, Robert R, Liao J, Matthes E, Carlile GW, Hanrahan JW, et al. Identification of a NBD1-binding pharmacological chaperone that corrects the trafficking defect of F508del-CFTR. Chem Biol. 2011; 18: 231-42.

59 Robert R, Carlile GW, Pavel C, Liu N, Anjos SM, Liao J, et al. Structural analog of sildenafil identified as a novel corrector of the F508delCFTR trafficking defect. Mol Pharmacol 2008; 73: 478-89.

60 Robert R, Carlile GW, Liao J, Balghi H, Lesimple P, Liu N, et al. Correction of the $\Delta \mathrm{F} 508$ cystic fibrosis transmembrane conductance regulator trafficking defect by the bioavailable compound glafenine. Mol Pharmacol. 2010; 77: 922-30.

61 Pedemonte N, Sonawane ND, Taddei A, Hu J, Zegarra-Moran O, et al. Phenylglycine and sulfonamide correctors of defective $\Delta \mathrm{F} 508$ and G551D cystic fibrosis transmembrane conductance regulator chloridechannel gating. Mol Pharmacol 2005; 67: 1797-807.

62 Pedemonte N, Boido D, Moran O, Giampieri M, Mazzei M, Ravazzolo R, et al. Structure-activity relationship of 1,4-dihydropyridines as potentiators of the cystic fibrosis transmembrane conductance regulator chloride channel. Mol Pharmacol 2007; 72: 197-207.

63 Van Goor F, Hadida S, Grootenhuis PD, Burton B, Cao D, Neuberger $\mathrm{T}$, et al. Rescue of CF airway epithelial cell function in vitro by a CFTR potentiator, VX-770. Proc Natl Acad Sci U S A 2009; 106: 18825-30.

64 Cai Z, Taddei A, Sheppard DN. Differential sensitivity of the cystic fibrosis (CF)-associated mutants G551D and G1349D to potentiators of the cystic fibrosis transmembrane conductance regulator (CFTR) $\mathrm{Cl}^{-}$channel. J Biol Chem 2006; 281: 1970-7.

65 Bompadre SG, Sohma Y, Li M, Hwang TC. G551D and G1349D, two CF-associated mutations in the signature sequences of CFTR, exhibit distinct gating defects. J Gen Physiol 2007; 129: 285-98.

66 Accurso FJ, Rowe SM, Clancy JP, Boyle MP, Dunitz JM, Durie PR, et al. Effect of VX-770 in persons with cystic fibrosis and the G551D-CFTR mutation. N Engl J Med 2010; 363: 1991-2003.

67 Ai T, Bompadre SG, Wang X, Hu S, Li M, Hwang TC. Capsaicin potentiates wild-type and mutant cystic fibrosis transmembrane conductance regulator chloride-channel currents. Mol Pharmacol 2004; 65: 1415-26.

68 Moran O, Galietta LJ, Zegarra-Moran O. Binding site of activators of the cystic fibrosis transmembrane conductance regulator in the nucleotide binding domains. Cell Mol Life Sci 2005; 62: 446-60.

69 Wang F, Zeltwanger S, Yang IC, Nairn AC, Hwang TC. Actions of genistein on cystic fibrosis transmembrane conductance regulator channel gating. Evidence for two binding sites with opposite effects. J Gen Physiol 1998; 111: 477-90. 
70 Zegarra-Moran O, Monteverde M, Galietta LJ, Moran O. Functional analysis of mutations in the putative binding site for cystic fibrosis transmembrane conductance regulator potentiators. Interaction between activation and inhibition. J Biol Chem 2007; 282: 9098 104.

71 Lansdell KA, Delaney SJ, Lunn DP, Thomson SA, Sheppard DN, Wainwright BJ. Comparison of the gating behaviour of human and murine cystic fibrosis transmembrane conductance regulator $\mathrm{Cl}^{-}$ channels expressed in mammalian cells. J Physiol 1998; 508: 379
92.

72 de Jonge $\mathrm{H}$, Wilke M, Bot A, Sheppard DN. Responsiveness of mouse versus human CFTR- $\Delta \mathrm{F} 508$ to small molecule correctors and potentiators. Pediatr Pulmonol Suppl 2009; 32: 291-92.

73 Scott-Ward TS, Cai Z, Dawson ES, Doherty A, Da Paula AC, Davidson

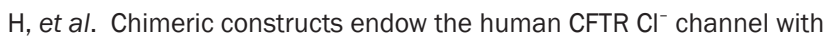
the gating behavior of murine CFTR. Proc Natl Acad Sci U S A 2007; 104: 16365-70. 